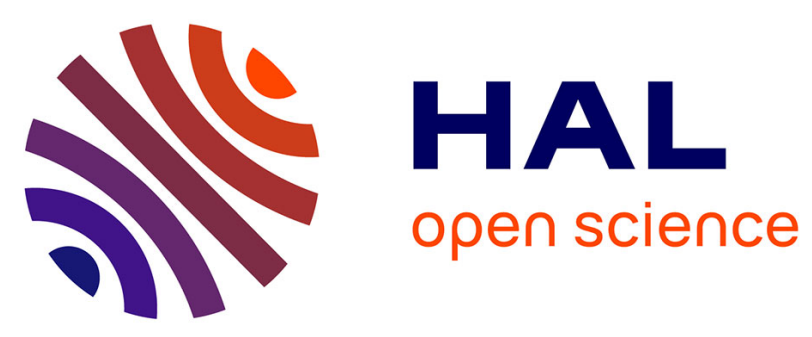

\title{
Simplified modeling of liquid sodium medium with temperature and velocity gradient using real thermal-hydraulic data. Application to ultrasonic thermometry in sodium fast reactor

\author{
N. Massacret, J. Moysan, M.A. Ploix, J. P. P Jeannot, G. Corneloup
}

\section{To cite this version:}

N. Massacret, J. Moysan, M.A. Ploix, J. P. P Jeannot, G. Corneloup. Simplified modeling of liquid sodium medium with temperature and velocity gradient using real thermal-hydraulic data. Application to ultrasonic thermometry in sodium fast reactor. Review of Progress in Quantitative Nondestructive Evaluation, 2012, Denver, United States. pp.1693-1700, 10.1063/1.4789245 . hal-01300004

\author{
HAL Id: hal-01300004 \\ https://hal.science/hal-01300004
}

Submitted on 27 Oct 2021

HAL is a multi-disciplinary open access archive for the deposit and dissemination of scientific research documents, whether they are published or not. The documents may come from teaching and research institutions in France or abroad, or from public or private research centers.
L'archive ouverte pluridisciplinaire HAL, est destinée au dépôt et à la diffusion de documents scientifiques de niveau recherche, publiés ou non, émanant des établissements d'enseignement et de recherche français ou étrangers, des laboratoires publics ou privés. 


\title{
SIMPLIFIED MODELING OF LIQUID SODIUM MEDIUM WITH TEMPERATURE AND VELOCITY GRADIENT USING REAL THERMAL-HYDRAULIC DATA. APPLICATION TO ULTRASONIC THERMOMETRY IN SODIUM FAST REACTOR
}

\author{
N. Massacret ${ }^{1}$, J. Moysan ${ }^{2}$, M. A. Ploix ${ }^{2}$, J. P. Jeannot ${ }^{1}$, and G. Corneloup ${ }^{2}$ \\ ${ }^{1}$ DEN/DTN/STPA/LIET, CEA Cadarache, Saint Paul Lez Durance, France \\ ${ }^{2}$ Aix-Marseille Univ, LMA UPR 7051 CNRS, site LCND, 13625 Aix-en-Provence, France
}

\begin{abstract}
In the framework of the French R\&D program for the Generation IV reactors and specifically for the sodium cooled fast reactors (SFR), studies are carried out on innovative instrumentation methods in order to improve safety and to simplify the monitoring of fundamental physical parameters during reactor operation. The aim of the present work is to develop an acoustic thermometry method to follow up the sodium temperature at the outlet of subassemblies. The medium is a turbulent flow of liquid sodium at $550^{\circ} \mathrm{C}$ with temperature inhomogeneities. To understand the effect of disturbance created by this medium, numerical simulations are proposed. A ray tracing code has been developed with $\mathrm{Matlab}^{\circ}$ in order to predict acoustic paths in this medium. This complex medium is accurately described by thermal-hydraulic data which are issued from a simulation of a real experiment in Japan. The analysis of these results allows understanding the effects of medium inhomogeneities on the further thermometric acoustic measurement.
\end{abstract}

Keywords: Ultrasonic, Thermometry, Sodium Fast Reactor, Acoustic Ray Simulation, Thermal Inhomogeneities.

\section{INTRODUCTION}

Within the framework of the French Act, dated June $28^{\text {th }} 2006$, which requests an assessment of the industrial perspectives of transmutation by 2012, the Generation IV systems, and especially the sodium-cooled fast reactors seem to be the most suitable technologies to be developed. Thus, since 2007, the CEA, EDF and AREVA French partners launched a coordinated research program on sodium cooled fast reactors (SFR) which is now being used for the next ASTRID (Advanced Sodium Technological Reactor for industrial Demonstration) prototype. The building of this last is planned on the horizon 2020 [1]. In this context the CEA (Atomic Energy Comission) must develop innovative and specific instrumentation to improve in particular the safety for this kind of reactor [2], and especially for liquid sodium which is an opaque fluid banning classical optical techniques. One of the objectives of ASTRID is to qualify for the SFR research program instrumentation methods. The measurement of sodium temperature coming out of the core is one of these methods. The current means for measuring temperature, the thermocouple 
included in a thermowell, presents some disadvantages in terms of precision and response time. So a new method is being developed using ultrasounds. To make this method reliable, studies have to be realized to understand and predict the propagation of ultrasound in a turbulent medium presenting high thermal inhomogeneities and strong speed gradients. After a short presentation of the current temperature measurement above a reactor, the acoustic method is presented and then how the propagation of ultrasound in this medium have been simulated. Lastly the perspective of some experiment will be described.

\section{TEMPERATURE MEASUREMENT ISSUES}

Temperature has to be measured just above each subassembly outlet. This information is important in order to get a map of sodium temperature and so monitoring several parameters as individual over-heating and different temperature threshold of subassemblies. This last surveillance allows also the detection of fast evolutions of temperature prevents from some potentially critical accidents able to lead to a partial or total fusion of the core.

The temperature is currently measured by thermocouples. However there are some disadvantages. First of all one thermowell must be disposed above each subassembly for which the output temperature has to be measured. Moreover there is a minimal distance between a thermocouple and a subassembly. This distance implies a possible influence of the neighboring subassemblies (in case of flow rate fluctuations in the different subassemblies) which could distort the measure. Finally the time response was about $4.5 \mathrm{~s}$ for Phénix and $1.1 \mathrm{~s}$ for Superphénix which should be improved for a continuous monitoring of the core.

To develop a new method of temperature measurement an important fact has to be considered: above the core the sodium is a highly turbulent medium; with lots of thermal inhomogeneities (the temperature difference between two subassemblies can reach $50{ }^{\circ} \mathrm{C}$ ), with strong velocity gradients (about $1.5 \mathrm{~m} . \mathrm{s}^{-1} \cdot \mathrm{cm}^{-1}$ ).

\section{ACOUSTIC THERMOMETRY METHOD}

This ultrasonic method was patented by the UKAEA (United Kingdom Atomic Energy Authority) in 1985 [3]. It is based on the fact that ultrasound celerity depends on sodium temperature. This is resumed by the Childs law [4]:

$$
c_{u s}=2577.25-0.524 \times \mathrm{T}
$$

where $c_{u s}$ represents the ultrasound celerity $\left(m \cdot s^{-1}\right), T$ is sodium temperature $\left({ }^{\circ} \mathrm{C}\right)$; The coefficients have been determined experimentally.

A monochromatic ultrasonic wave propagates with grazing incidence to a subassembly. Then the transducer receives two echoes, coming from the reflection on the edges of the subassembly. Knowing the distance between these edges and measuring the time of flight of the ultrasounds separating the two, the ultrasound celerity in this area can be calculated and thus the sodium temperature can be estimated with the Childs law. A schema on Fig. 1 represents this method. 


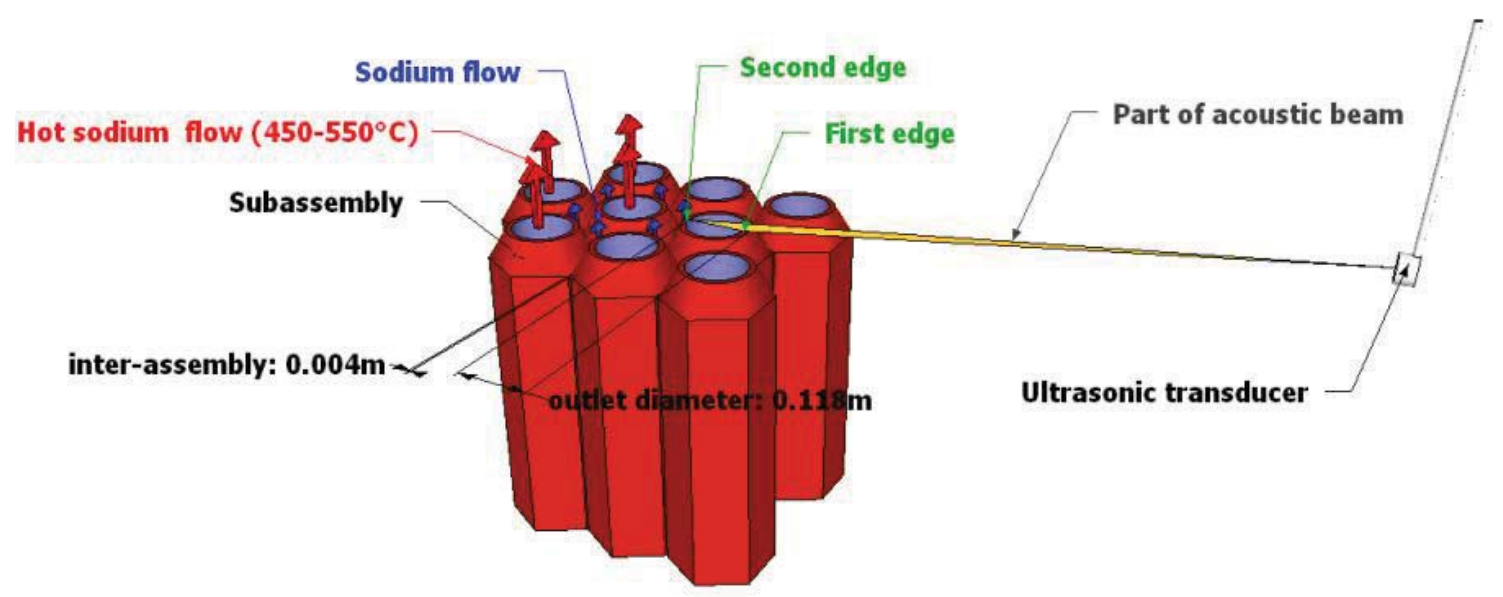

FIGURE 1. Representation of the acoustic method.

This method provides several advantages:

- The response time is mainly limited by the signal processing; thus it is possible to have a frequency response of several kilohertz.

- This measurement is local because only the sodium temperature between the edges of a subassembly is measured. There is no more influence of the neighboring subassemblies.

- Finally this is a non-invasive method which requires fewer devices supposing it is theoretically possible to measure the temperature of a row of subassemblies with only one transducer.

However the propagation of ultrasound in sodium may be difficult to predict in presence of temperature and speed flow inhomogeneities. A local difference of temperature will change the ultrasound celerity and so will modify the wave-front. Important temperature will also change the wave propagation direction. Moreover strong speed flow gradient may have the same influence on the ultrasounds. Thus, all these phenomena will contribute to deflect the ultrasonic beam. The risks for the acoustic thermometry are a possible interruption of the measurement, or in the worst case, the measurement of another subassembly temperature would fool the monitoring.

This emphasizes the importance of understanding and predicting the wave propagation in such a turbulent medium. A simulation tool based on the acoustic ray theory has been developed to carry out this study.

\section{ACOUSTIC SIMULATION}

\section{Propagation Medium}

There are few existing data regarding sodium flowing out of the subassemblies and most of the existing studies describe the medium with a large scale that is not compatible with the acoustic propagation scale. Indeed, the wave length of a $1 \mathrm{MHz}$ acoustic wave in sodium at $550{ }^{\circ} \mathrm{C}$ is about $2.3 \mathrm{~mm}$, the scale of the thermal-hydraulic data must be of the same order. 
The thermal-hydraulic data chosen for this work come from a JAEA (Japan Atomic Energy Agency) experiment named PLAJEST [5], where a tree of thermocouples measures the amplitude and the frequency of temperature fluctuations, of three hot and cold sodium jets. This experiment was then simulated very precisely with the CEA thermal-hydraulic code TRIO-U in order to check the validity of the simulation model $[5,6]$.

This simulation provides the temperature of the sodium and the speed flow vector at a very precise scale: the characteristic length of the mesh is about two or three millimeters. The very short time step of $0.01 \mathrm{~s}$ enables to see all the thermal-hydraulic phenomena. On the one hand the experimental geometry is close to a row of subassemblies, on the other hand the agreement between simulation data and experimental data was rather good (see [6]), so this simulation is a good set of data to evaluate ultrasound propagation above core of sodium fast reactor.

\section{Acoustic Ray Tracing Method}

This method enables to determine all the successive position of one point of the wave front, drawing in this way an acoustic ray (also named acoustic path). Thanks to this method it's possible to compute the deviation of ultrasounds propagating through a complex medium, as well as caustics (area where an ultrasound beam is focusing). It is an iterative calculation which considers for each step local thermal-hydraulic data for each step.

The variation of propagation direction is calculated by using a refractive index based on temperature and velocity speed fields as it appears in the acoustic ray equations (2) [7]:

$$
\left\{\begin{array}{c}
\frac{d \vec{r}}{d s}=c \vec{S}+\frac{\Omega}{c} \vec{v} \\
\frac{d \vec{S}}{d s}=-\frac{\Omega^{2}}{c^{2}} \nabla c-\frac{\Omega}{c} \sum_{i=x, z} S_{i} \nabla v_{i} \\
\frac{d \tau}{d s}=\frac{\Omega}{c}
\end{array}\right.
$$

where $\vec{r}(x, z)$ is the $2 \mathrm{D}$ ray position vector, $s$ is the arc length, $\tau(r)$ is the travel time of the wave on the ray, $c(r)$ is the acoustic velocity, $\vec{v}(r)$ the fluid velocity vector, $\vec{S}=\frac{\vec{t}}{c+\vec{t} . \vec{v}}$ is the slowness vector, $\vec{t}(r)$ is the unit vector tangent to the ray and $\Omega=1-\vec{v} \cdot \vec{S}$.

One of the difficulties to couple this model with the thermal-hydraulic data is that these data are only given for the mesh center of the TRIO-U simulation whereas the ray tracing method needs data in the whole space. An interpolation method using a Delaunay triangulation is developed. It enables to select the three data points between which a point of the ray is situated. A linear approximation is performed with these three data points in order to attribute thermal-hydraulic values to the point of the ray.

\section{$\underline{\text { Acoustic Ray Simulations }}$}

The first computation simulates the 2D-propagation of several acoustics rays through a vertical plan of PLAJEST data. In this simulation the rays start from the edges of the sodium jets and focus at a point outside of the jets at an elevation of $2 \mathrm{~cm}$. The main goal of this simulation is to propagate rays across sodium jets presenting different temperatures to estimate the influence of thermal-hydraulic phenomena in a simple case. 
The geometry of this simulation corresponds to the propagation of rays above a core in a vertical plan passing through the axe of a row of subassemblies, at a smaller scale (here the jet diameter is about $2 \mathrm{~cm}$, whereas in the real case the subassemblies diameters are about $10 \mathrm{~cm}$ ). The caustic corresponds to the position of a transducer.

At first rays are propagated without considering the temperature and velocity gradient influence in order to check the focusing of the rays. In the second case, thermalhydraulic data influence the propagation of the rays and deflect them. The results of the simulation are presented on Fig. 2.

The ultrasonic deviation caused by temperature and velocity is finally small in this configuration, where gradients are collinear to the wave propagation direction. It appears that the beam width at the focus point only reaches $0.5 \mathrm{~mm}$.

Thanks to this result it can be deduced that the part of the beam which propagates in the axe of a subassemblies row will not suffer from an important vertical deviation. It is an important result for the thermometry method because if the beam is deflected between the two edges, the acoustic path will not stay as a line and the travelled distance will change. As a result, the temperature measures will not be accurate.

The next simulation computes a ray propagating horizontally through the three sodium jets. The aim is to distinguish temperature influence from velocity influence. In a first case calculations consider both temperature and speed flow influence, in the second case only the temperature influence. As the distance of sound propagation $(2 \mathrm{~cm}$ for each jet compared to $10 \mathrm{~cm})$ is small as well as the value of the vertical velocity $\left(0.5 \mathrm{~m} . \mathrm{s}^{-1}\right.$ compared to $3 \mathrm{~m} . \mathrm{s}^{-1}$ ), in the three jets compared to a real case the choice was made to multiply the value of the vertical velocity by ten. The comparison of the two results with and without flow is presented on Fig. 3.
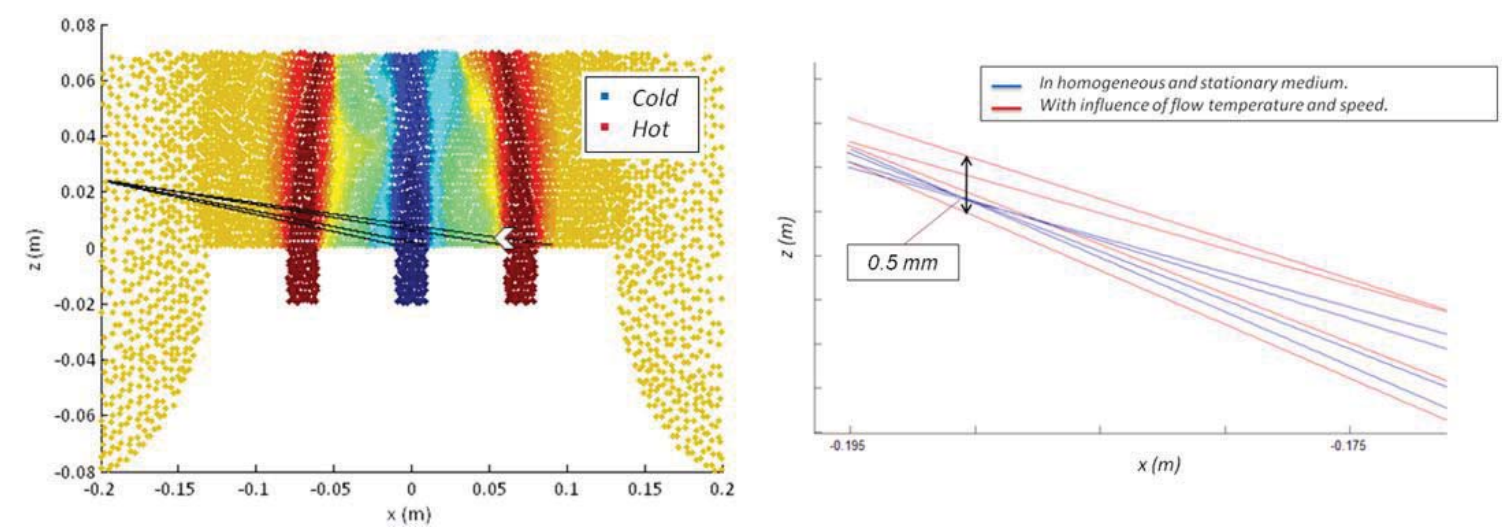

FIGURE 2. Ray propagation across three sodium jets (left) and deflection of the focusing beam (right) when considering thermal-hydraulic phenomena.

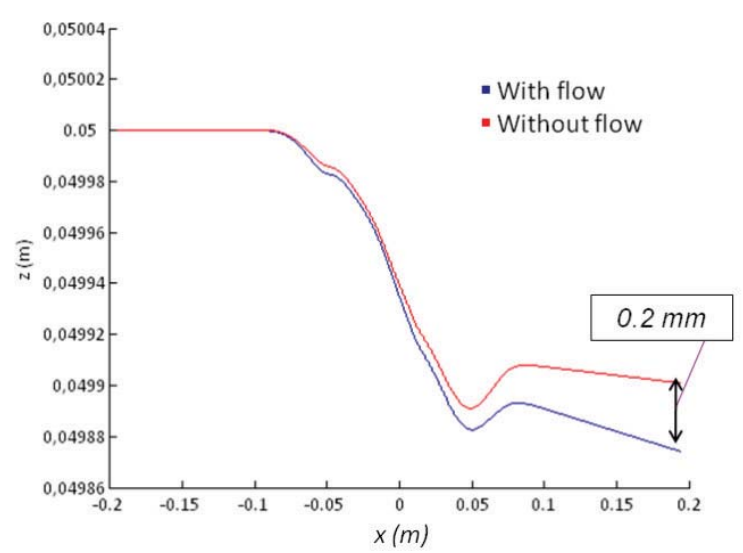

FIGURE 3. Influence of flow speed on acoustic path deviation. 
Results show that influence of velocity gradient is, on the one hand very small because deviation engendered is only about $0.2 \mathrm{~mm}$, effect on the wave propagation five times smaller than the influence of temperature.

However because of recirculation phenomena between real subassemblies, some eddies might be generated on the path of the acoustic beam. That is the reason why more complex speed flow gradients have to be computed before concluding on the influence of speed flow.

The PLAJEST data were extrapolated in order to consider the effects of temperature gradients in a reactor, with multiple assemblies. Only the Gaussian temperature gradient between the steady state medium and a hot sodium jet was kept. Then a new map of temperature was created with some rotations and translations, corresponding to the temperature field of a horizontal plan above three subassemblies. This map, including the original PLAJEST data, is represented on Fig. 4.

Here several parallel rays are propagated from the left to the right. As the wave direction propagation is almost orthogonal to the temperature gradient, the deviation is in this case more important than previously: as presented in Fig. 5, the deviation exceeds $1 \mathrm{~cm}$ after 60 $\mathrm{cm}$ of propagation.

This deviation is not important enough to miss the monitored subassembly. However it could have other significant effects. In this case the deviated rays could have an orthogonal incidence on the edges of subassemblies and so return a signal to the transducer. They propagate through a medium in which the ultrasound celerity is different. This implies that their time of flight will be different from the direct ray, and that the signal received by the transducer will be noised.

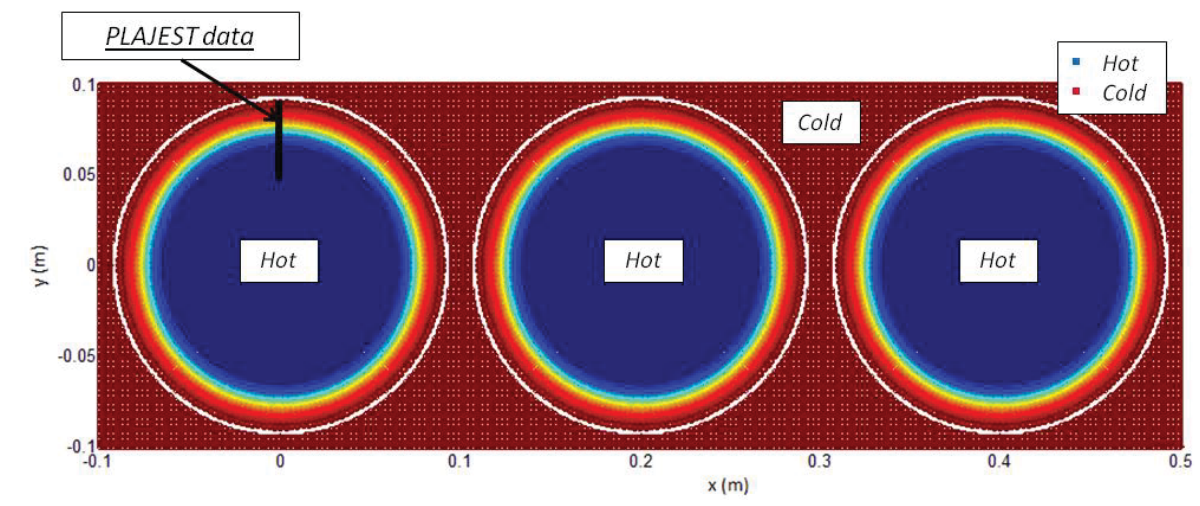

FIGURE 4. Temperature map of a row of three subassemblies.

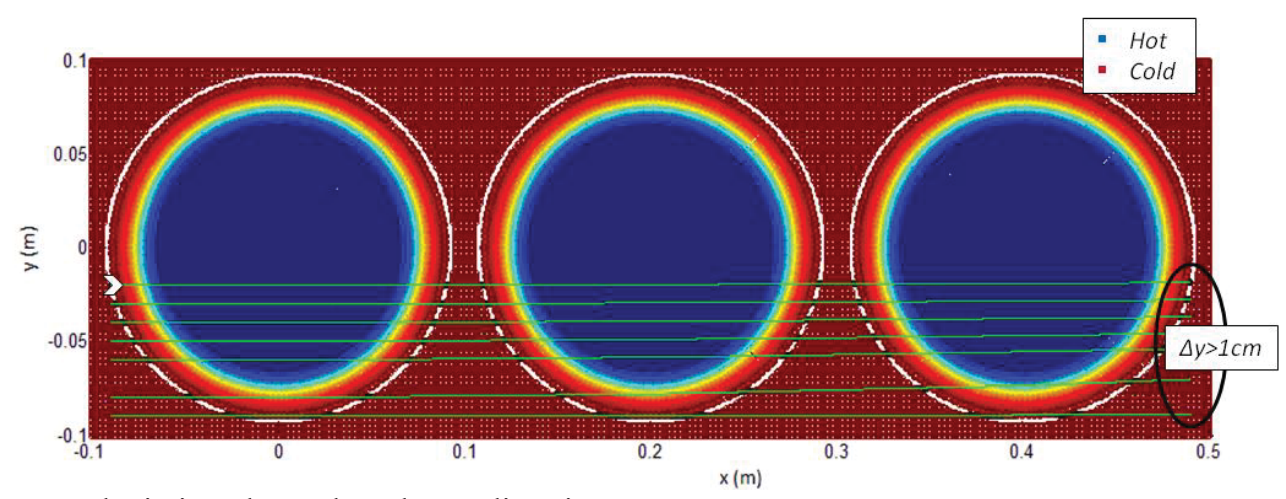

FIGURE 5. Ray deviation above three hot sodium jets. 
Further work on this kind of simulation will be performed to determine the direction of deflection and the time of flight of these rays. The aim is, to evaluate the impact on the thermometry and to enable the design of future transducers.

\section{Further Developments and Conclusions}

Further work will bring some improvement to the simulations. First of all Gaussian beam method will be developed [7]; this method enables the calculation of the acoustic pressure in all the points of the geometry and not only along the calculated acoustic paths. Thus the calculation of the signal amplitude in caustics or in shadowed areas will be more precisely.

In order to check the validity of this simulation tool, two experiments are planned:

- The first one will analyze the propagation of ultrasounds in a thermal inhomogeneous medium. A fluid of high viscosity, silicon oil, will be used to limit convection phenomena and to realize acoustic measurements in a quite stationary medium. A schlieren strioscopic bench will be used to realize correlation between thermal and acoustic data.

- The second one will enable to propagate ultrasounds in a medium with high speed gradients. As it is difficult to know the thermal-hydraulic data of such a medium at any time (in order to realize correlation between acoustic and thermal-hydraulic data), instabilities of Kelvin-Helmholtz will be created. These instabilities are well known and allow to have precise thermal-hydraulic data on eddies where ultrasounds propagate.

Finally this ray tracing method will be compared with another numerical method: the SPECFEM code which uses the spectral element method [8]. It is highly parallelizable and could be a solution to simulate the ultrasounds propagation important volumes (several cubic meters).

All these developments demonstrate that ultrasonic thermometry could be evaluated precisely for future in service monitoring.

\section{ACKNOWLEDGEMENTS}

Authors would like to thank the Trio-U thermal-hydraulic team from the CEAGrenoble for their support in transmitting numerical data of the PLAJEST simulation.

\section{REFERENCES}

1. P. Philippon, Les défis du CEA 152, «Astrid, le projet à réaction » (2010).

2. F. Jadot, F. Baqué, J.Ph. Jeannot, G. de Dinechin, J.M. Augem, J. Sibilo, “ASTRID Sodium cooled Fast Reactor: Program for improving In Service Inspection and Repair", ANIMMA conf., Ghent, Belgium, Paper 3.37 (2011).

3. J. A. McKnight, L. D. Macleod, E. J. Burton, US Patent No. 8,402,920 (February 4, 1985).

4. WHJ Childs, Physical constants, Chapman \& G. Hall, 1972.

5. N. Kimura et al., International Journal of Heat and Mass Transfer 50 (2007) pp. 2024-2036.

6. D. Tenchine, Nuclear Engineering and Design 240 (2010) pp 1195-1217.

7. B. Iooss and al., Ultrasonics 40 (2002) pp. 1009-1015.

8. D. Komatitsch, J.P. Violette., "The spectral -element method: an efficient tool tosimulate the seismic response of 2D and 3D geological structures", Bull. Seismol. Soc. Am. 88 (1998) pp.368-392. 
Copyright of AIP Conference Proceedings is the property of American Institute of Physics and its content may not be copied or emailed to multiple sites or posted to a listserv without the copyright holder's express written permission. However, users may print, download, or email articles for individual use. 